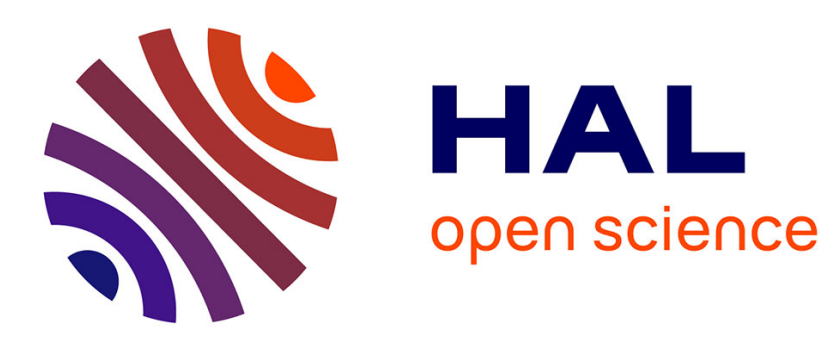

\title{
A Transmission Quality Index for a Class of Four-limb Parallel Schönflies Motion Generators
}

\author{
Guanglei Wu, Shaoping Bai, Stéphane Caro
}

\section{To cite this version:}

Guanglei Wu, Shaoping Bai, Stéphane Caro. A Transmission Quality Index for a Class of Four-limb Parallel Schönflies Motion Generators. Journal of Mechanisms and Robotics, 2018, 10 (5), pp.051014. 10.1115/1.4040353. hal-01860812

\section{HAL Id: hal-01860812 \\ https://hal.science/hal-01860812}

Submitted on 23 Aug 2018

HAL is a multi-disciplinary open access archive for the deposit and dissemination of scientific research documents, whether they are published or not. The documents may come from teaching and research institutions in France or abroad, or from public or private research centers.
L'archive ouverte pluridisciplinaire HAL, est destinée au dépôt et à la diffusion de documents scientifiques de niveau recherche, publiés ou non, émanant des établissements d'enseignement et de recherche français ou étrangers, des laboratoires publics ou privés. 


\title{
A Transmission Quality Index for a Class of Four-limb Parallel Schönflies Motion Generators
}

\author{
Guanglei $\mathrm{Wu}^{1}$, Shaoping Bai ${ }^{2}$, Stéphane Caro ${ }^{3}$ \\ ${ }^{1}$ School of Mechanical Engineering, Dalian University of Technology, 116024 Dalian, China \\ E-mail: gwu@dlut.edu.cn \\ ${ }^{2}$ Department of Mechanical and Manufacturing Engineering, Aalborg University, 9220 Aalborg, Denmark \\ E-mail: shb@mp.aau.dk \\ ${ }^{3}$ CNRS, Laboratoire des Sciences du Numérique de Nantes, UMR CNRS 6004 \\ Ecole Centrale de Nantes, 44321 Nantes, France \\ E-mail: stephane.caro@ls2n.fr
}

\begin{abstract}
This paper presents a uniform evaluation method for both transmission quality and singularity analysis for a class of parallel Schönflies-motion generators with four $R R \Pi R R$ limbs. It turns out that the determinant of the forward Jacobian matrices for this class of parallel robots can be expressed as the scalar product of two vectors, the first vector being the cross product of the four unit vectors along the parallelograms, and the second one being related to the rotation of the mobile platform. The pressure angles, derived from the determinants of forward and inverse Jacobians, respectively, are used for the evaluation of the transmission quality and the detection of robot singularities. Five robots are compared based on the proposed indices as an illustrative example.
\end{abstract}

Keywords: Schönflies motion, pick-and-place, transmission index, pressure angle, singularity

\section{Introduction}

Parallel robots performing Schönflies motions or SCARA (Selective Compliance Assembly Robot Arm) motion [1], namely, three independent translations and one rotation about an axis of fixed direction (3T1R), are well adapted to high-speed pick-and-place (PnP) operations, thanks to their advantages in terms of lightweight structure and high stiffness. Amongst those parallel Schönflies-motion generators 
(SMGs) [2-6], most of them inherit the architecture of the H4 [7] robot with four identical limbs and an articulated traveling plate [8]. Its similar version is the I4 robot [9] and the symmetrical Par4 [10]. The commercial version of Par4, namely, the Quattro robot [11] by Adept Technologies Inc., is the fastest industrial robot available. The rotation is realized by the relative movement in parallel between the two sub-platforms in this double-platform architecture, and the rotation can also be amplified by the pulley-belt mechanism [11]. This articulated system enhances the rotational capability of the robot end-effector. However, the manufacturing cost is getting more expensive. Therefore, if the applications do not require a high rotational capability, the end-effector of the robot free of the amplification mechanism is preferable to simplify the robot architecture. Later on, the Veloce. robot [12] with a different articulated traveling plate that is connected by a screw pair, was developed. Besides, the four-limb robots with single-platform architecture have also been reported [13-15]. Other parallel Schönflies-motion robots are also noticeable for their high performance [16-20], such as the Delta based robots [21] and the two-limb SMGs [22-25], etc.. The four-limb parallel robots with an articulated mobile platform are displayed in Fig. 1. It is noteworthy that the H4 robot with the modified mobile platform can be mounted vertically instead of the horizontal installation with reduced mounting space [26], in order to provide a rotation around an axis of vertical direction, which is named as "V4" for convenience in the following study.

In the design and application of a manipulator, its kinematic Jacobian matrix plays an important role, since the dexterity/manipulability of the robot can be evaluated through the conditioning number of the kinematic Jacobian matrix. This quantity also reflects the accuracy/wrench capability [27] between the actuators and end-effector. On the other hand, in the performance evaluation using the Jacobian matrix, a problem usually encountered is that the parallel manipulators with mixed input or/and output motions, i.e., compound linear and angular motions, will result in dimensionally inhomogeneous Jacobians. Thus, the performance indices of the conventional Jacobian matrix, such as condition number, will lack in physical significance [28]. As far as SMGs are concerned, their endeffector generates a mixed motion, i.e., the Schönflies motions, for which the terms of the kinematic Jacobian matrix do not have the same units. The common approach to overcome this problem is to introduce a characteristic length $[29,30]$ to homogenize the Jacobian matrix or the formulation of the point-based Jacobian matrices $[31,32]$ by means of vertex velocity transformation of the mobile platform. On the other hand, the measurement significantly depends on the choice of the characteristic length or the selection of the vertices that is not unique, which results in biased evaluation, although a "best" one can be found by optimization technique [33]. Alternatively, an efficient approach to ac- 


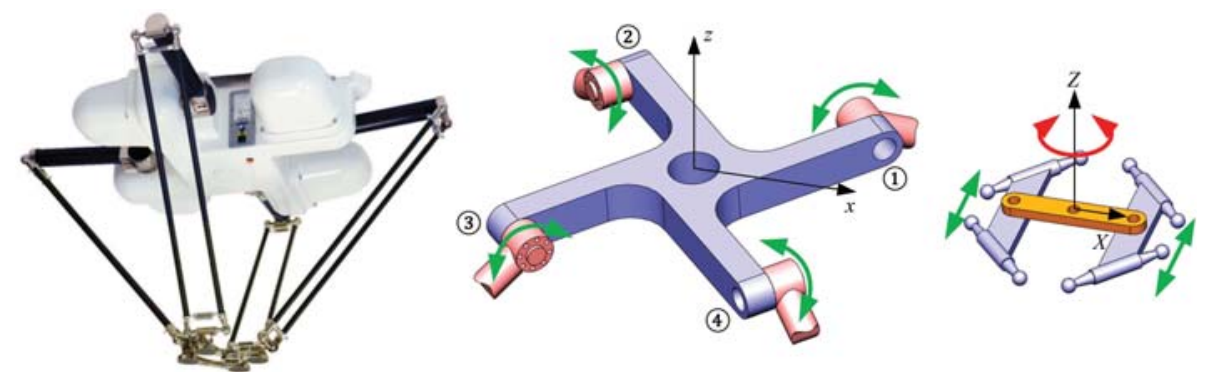

(a)
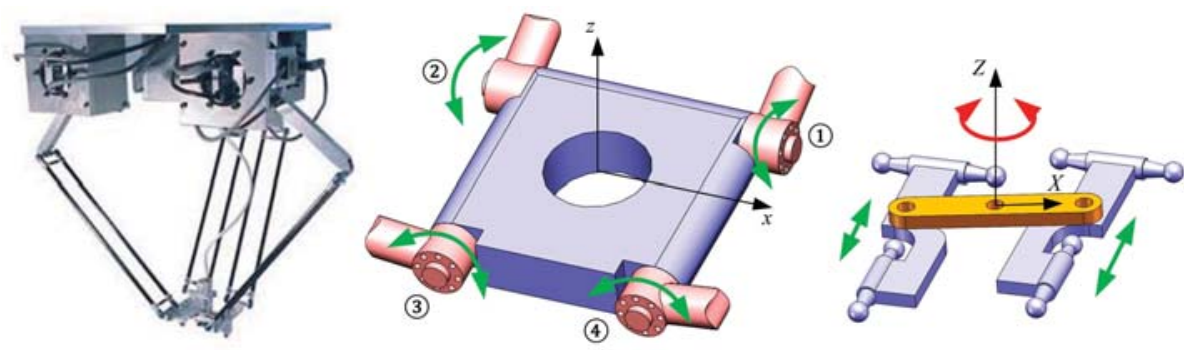

(b)
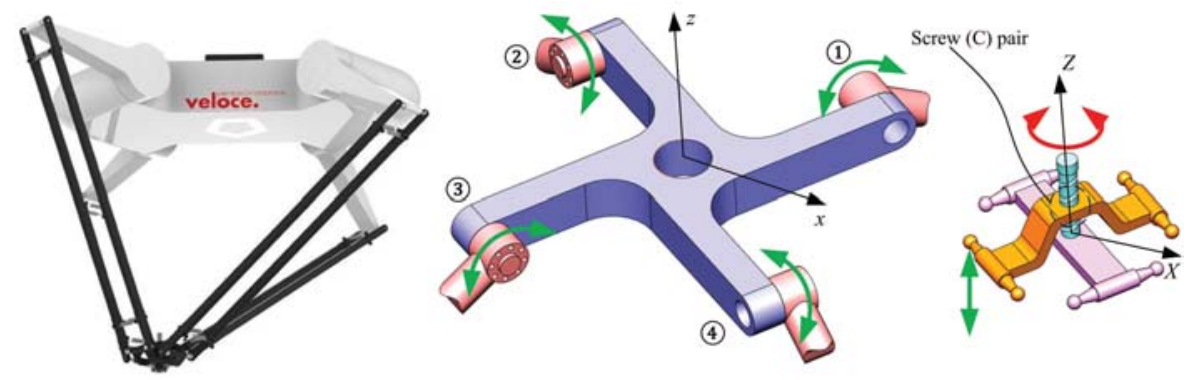

(c)
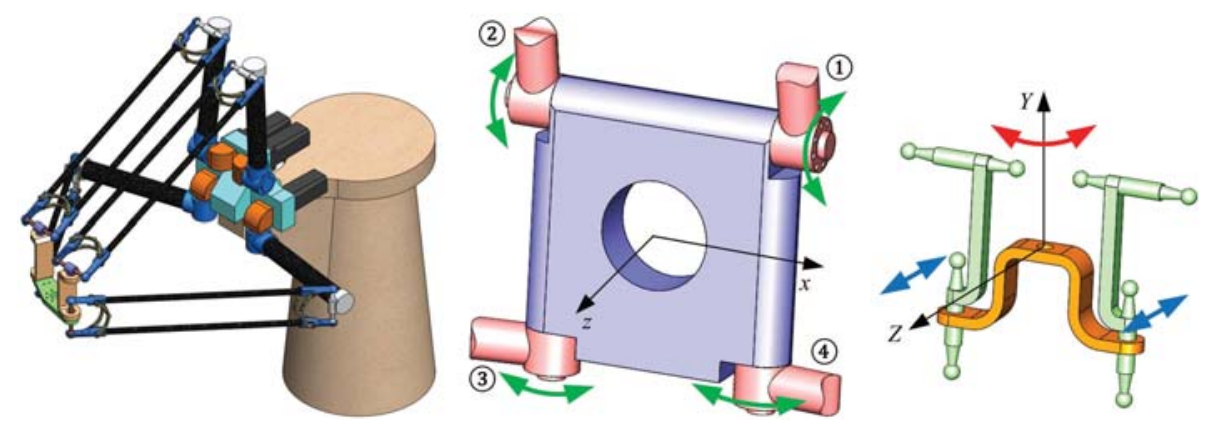

(d)

Figure 1: The four-limb parallel $\mathrm{PnP}$ robots with different base and mobile platform shapes: (a) Quattro [11]; (b) H4 [7]; (c) Veloce. [12]; (d) "V4" [26]. 
commodate this dimensional inhomogeneity is to adopt the concept of the virtual coefficient, namely, the transmission index, which is closely related to the transmission/pressure angle [34-37]. The screw theory based transmission indices have been widely used to evaluate the motion/force transmissibility of various parallel manipulators [38-42]. Moreover, the analysis of singularities is a central issue due to their major effect onto the robot performance. The transmission index being bounded between 0 and 1 and characterized by the sine/cosine function of the transmission/pressure angle, the transmission analysis can be used to identify singularities numerically and to evaluate the closeness to a singularity [43], compared to the Grassmann geometry, Grassman-Cayley algebra and the Jacobian method [44-47]. Accordingly, the pressure angle is adopted as the transmission index in this work.

This paper presents a uniform evaluation approach for transmission quality and singularity analysis of a family of four-limb 3T1R parallel robots with an articulated mobile platform. We show that the determinant of the forward Jacobian matrices for this class of parallel robots can be expressed as the scalar product of two vectors, the first vector being the cross product of the four unit vectors along the parallelograms, the second one being related to the rotation of the mobile platform. The pressure angles, derived from the forward and inverse Jacobians straightforward, are used for both the evaluation of the transmission quality and the singularity identification of the robots. Finally, five parallel Schönflies motion generators are compared based on the proposed index as an illustrative example.

\section{Manipulator Architecture}

Figure 2(a) depicts the simplified CAD model of a Schönflies-motion robot, which is composed of

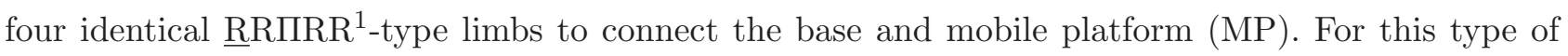
robots, the universal (U) joints composed of $\Pi$ joints is kinematically equivalent to parallelogram with spherical (S) joints, hence, the $\mathrm{U}$ joints are adopted to depict the robot linkage. The general base platform and the different mobile platforms are displayed in Figs. 2(b) and 2(c), respectively.

The fixed coordinate frame $\mathcal{F}_{b}$ is built with the origin located at the geometric center of the base platform. The $x$-axis is parallel to the segment $A_{2} A_{1}\left(A_{3} A_{4}\right)$, and the $z$-axis is normal to the baseplatform plane pointing upwards. The moving coordinate frame $\mathcal{F}_{p}$ is attached to the mobile platform and the origin is at the geometric center, where $X$-axis is parallel to segment $C_{2} C_{1}\left(C_{3} C_{4}\right)$. Hereafter, vectors $\mathbf{i}, \mathbf{j}$ and $\mathbf{k}$ represent the unit vectors along $x$-, $y$ - and $z$-axis, respectively. The axis of rotation

\footnotetext{
${ }^{1} \mathrm{R}$ and $\Pi$ stand for revolute joint and parallelogram (П) joint, respectively. The underlined letter indicates an actuated joint.
} 


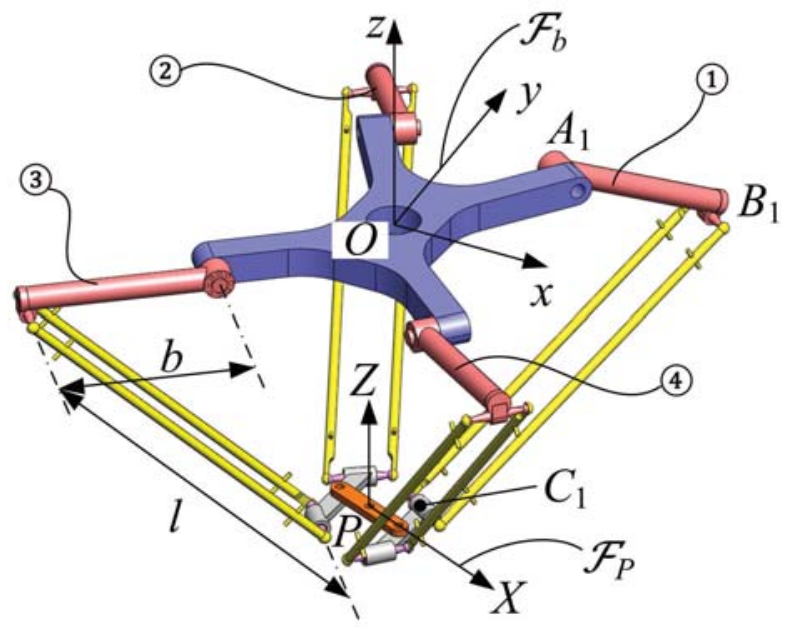

(a)

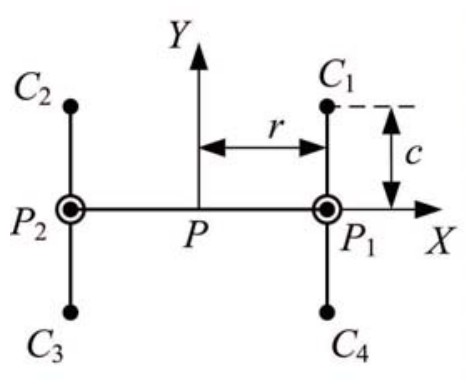

Quattro, H4

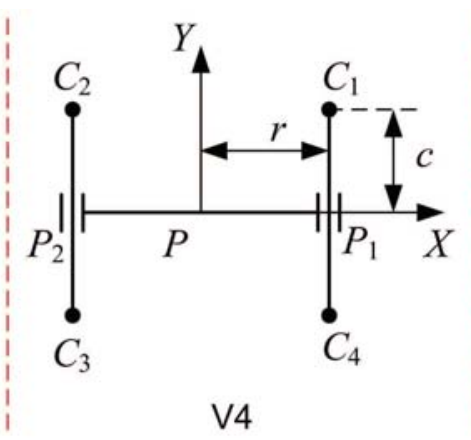

(c)

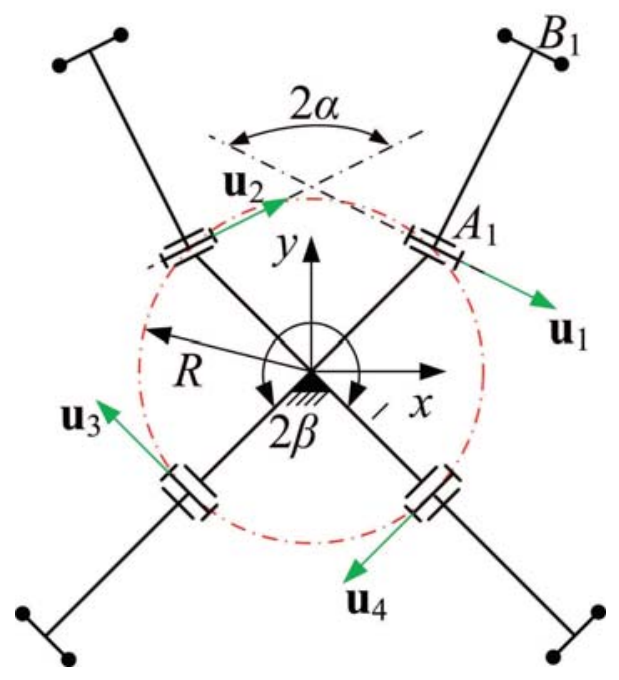

(b)

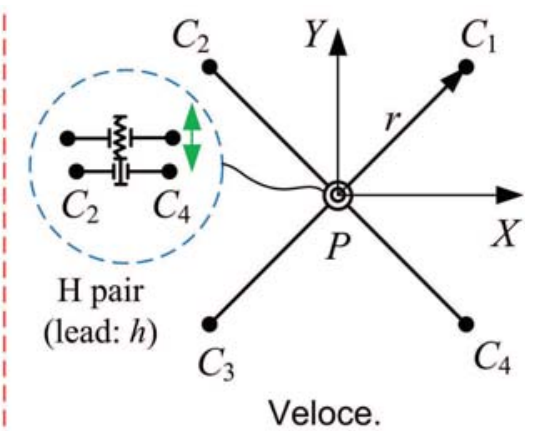

Veloce.

Figure 2: The parameterization of the four-limb 3T1R parallel robots: (a) simplified CAD model; (b) a general base platform; (c) different mobile platforms. 
Table 1: Cartesian coordinates of points $C_{i}$ and unit vectors of $\mathbf{s}_{i}{ }^{a}$

\begin{tabular}{lll}
\hline \hline robots & $\mathbf{c}_{i}$ & $\mathbf{s}_{i}$ \\
\hline Quattro, H4 & $\operatorname{sgn}\left(\cos \eta_{i}\right) r \mathbf{R}_{z}(\phi) \mathbf{i}+\operatorname{sgn}\left(\sin \eta_{i}\right) c \mathbf{j}+\mathbf{p}$ & $\operatorname{sgn}\left(\cos \eta_{i}\right) r \mathbf{s}, \mathbf{s}=\mathbf{R}_{z}(\phi) \mathbf{j}$ \\
V4 & $-\operatorname{sgn}\left(\cos \eta_{i}\right) r \mathbf{R}_{y}(\phi) \mathbf{i}+\operatorname{sgn}\left(\cos \eta_{i}\right) c \mathbf{j}+\mathbf{p}$ & $\operatorname{sgn}\left(\cos \eta_{i}\right) r \mathbf{s}, \mathbf{s}=\mathbf{R}_{y}(\phi) \mathbf{k}$ \\
Veloce. & $r \mathbf{R}_{z}\left(\eta_{i}\right) \mathbf{i}+\bmod (i, 2) h \phi /(2 \pi) \mathbf{k}+\mathbf{p}$ & $\bmod (i, 2) h \phi /(2 \pi) \mathbf{k}$ \\
\hline \hline
\end{tabular}

${ }^{a} \operatorname{sgn}(\cdot)$ stands for the sign function of $(\cdot)$, and mod stands for the modulo operation, $h$ being the lead of the screw pair of the Veloce. robot

of the $i$ th actuated joint is parallel to unit vector $\mathbf{u}_{i}=\mathbf{R}_{z}\left(\alpha_{i}\right) \mathbf{i}$, where $\mathbf{R}$ stands for the rotation matrix around an axis, and $\alpha_{1}=-\alpha_{2}=\alpha, \alpha_{3}=-\alpha_{4}=\beta$. Moreover, unit vectors $\mathbf{v}_{i}$ and $\mathbf{w}_{i}$ are parallel to segments $\overline{A_{i} B_{i}}$ and $\overline{B_{i} C_{i}}$, respectively, namely, the unit vectors along the active link and parallelogram in the $i$ th limb.

\section{Kinematics and Jacobian Matrices of the Robots}

The Cartesian coordinates of points $A_{i}$ and $B_{i}$ expressed in the frame $\mathcal{F}_{b}$ are respectively derived by

$$
\begin{aligned}
& \mathbf{a}_{i}=R\left[\begin{array}{lll}
\cos \eta_{i} & \sin \eta_{i} & 0
\end{array}\right]^{T} \\
& \mathbf{b}_{i}=b \mathbf{v}_{i}+\mathbf{a}_{i} ; \mathbf{v}_{i}=\mathbf{R}_{z}\left(\alpha_{i}\right) \mathbf{R}_{x}\left(\theta_{i}\right) \mathbf{j}
\end{aligned}
$$

where $\eta_{i}=(2 i-1) \pi / 4, i=1, \ldots, 4$, and $\theta_{i}$ are the input angles.

Let the mobile platform pose be denoted by $\boldsymbol{\chi}=\left[\begin{array}{ll}\mathbf{p}^{T} & \phi\end{array}\right]^{T}, \mathbf{p}=\left[\begin{array}{lll}x & y & z\end{array}\right]^{T}$. The inverse geometric problem of the manipulators is presented in detail in [2]. It can be solved from the following kinematic constraint equations:

$$
\left(\mathbf{c}_{i}-\mathbf{b}_{i}\right)^{T}\left(\mathbf{c}_{i}-\mathbf{b}_{i}\right)=l^{2}, \quad i=1, \ldots, 4
$$

where $\mathbf{c}_{i}$ are the Cartesian coordinates of point $C_{i}$ in frame $\mathcal{F}_{b}$, as listed in Table 1.

Let $\mathbf{w}_{i}=\left(\mathbf{c}_{i}-\mathbf{b}_{i}\right) / l$, upon differentiating Eq. (3) with respect to time, one obtains the following equation

$$
\mathbf{w}_{i}^{T}\left(\dot{\mathbf{c}}_{i}-\dot{\mathbf{b}}_{i}\right)=\mathbf{w}_{i}^{T}\left(\dot{\phi} r \mathbf{s}_{i}+\dot{\mathbf{p}}-\dot{\theta}_{i} b \mathbf{u}_{i} \times \mathbf{v}_{i}\right)=0
$$

that can be expressed as

$$
\mathbf{w}_{i}^{T} \dot{\mathbf{p}}+\dot{\phi} r \mathbf{w}_{i}^{T} \mathbf{s}_{i}=\dot{\theta}_{i} b \mathbf{w}_{i}^{T}\left(\mathbf{u}_{i} \times \mathbf{v}_{i}\right), \quad i=1, \ldots, 4
$$


where $\dot{\mathbf{p}}=\left[\begin{array}{lll}\dot{x} & \dot{y} & \dot{z}\end{array}\right]^{T}$, and $\mathbf{s}_{i}$ are unit vectors that depend on the rotation angle of the mobile platform given in Table 1. Equation (5) can be cast in a matrix form:

$$
\mathbf{A} \dot{\chi}=\mathbf{B} \dot{\theta}
$$

with

$$
\begin{aligned}
& \mathbf{A}=\left[\begin{array}{llll}
\mathbf{e}_{1} & \mathbf{e}_{2} & \mathbf{e}_{3} & \mathbf{e}_{4}
\end{array}\right]^{T} ; \quad \dot{\boldsymbol{\chi}}=\left[\begin{array}{llll}
\dot{x} & \dot{y} & \dot{z} & \dot{\phi}
\end{array}\right]^{T} \\
& \mathbf{B}=\operatorname{diag}\left[\begin{array}{llll}
h_{1} & h_{2} & h_{3} & h_{4}
\end{array}\right] ; \quad \dot{\boldsymbol{\theta}}=\left[\begin{array}{llll}
\dot{\theta}_{1} & \dot{\theta}_{2} & \dot{\theta}_{3} & \dot{\theta}_{4}
\end{array}\right]^{T}
\end{aligned}
$$

where $\mathbf{A}$ and $\mathbf{B}$ are the forward and inverse Jacobian matrices, respectively, and

$$
\mathbf{e}_{i}=\left[\begin{array}{ll}
\mathbf{w}_{i}^{T} & r \mathbf{w}_{i}^{T} \mathbf{s}_{i}
\end{array}\right]^{T} ; \quad h_{i}=b \mathbf{w}_{i}^{T}\left(\mathbf{u}_{i} \times \mathbf{v}_{i}\right)
$$

As long as $\mathbf{A}$ is nonsingular, the kinematic Jacobian matrix of the manipulators is expressed as

$$
\mathbf{J}=\mathbf{A}^{-1} \mathbf{B}
$$

For a given pose of the moving-platform, each limb can have two postures, which are characterized by the sign " $-/+$ " of $h_{i}$. It means that the robot can have up to sixteen working modes, i.e., sixteen solutions to the inverse kinematics problem. In order for the robot not to reach any serial singularity and to remain in one working mode, the working mode defined by $h_{i}<0, i=1, \ldots, 4$, is selected. The robot is supposed not to switch from this working mode to another one.

\section{Performance Evaluations}

Our interests are the transmission quality and also the singularity, both are related to the robot Jacobian $[26]$.

\subsection{Transmission Quality}

Here, two pressure angles, corresponding to the motion (input) and force (output) transmissions, are first derived from the forward and inverse Jacobians, respectively. 


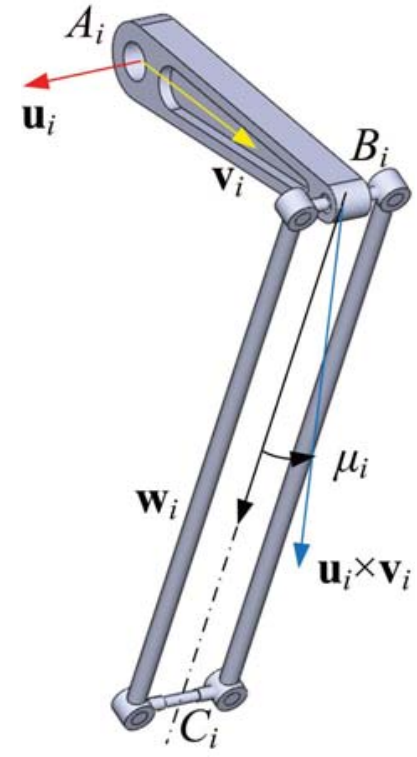

(a)

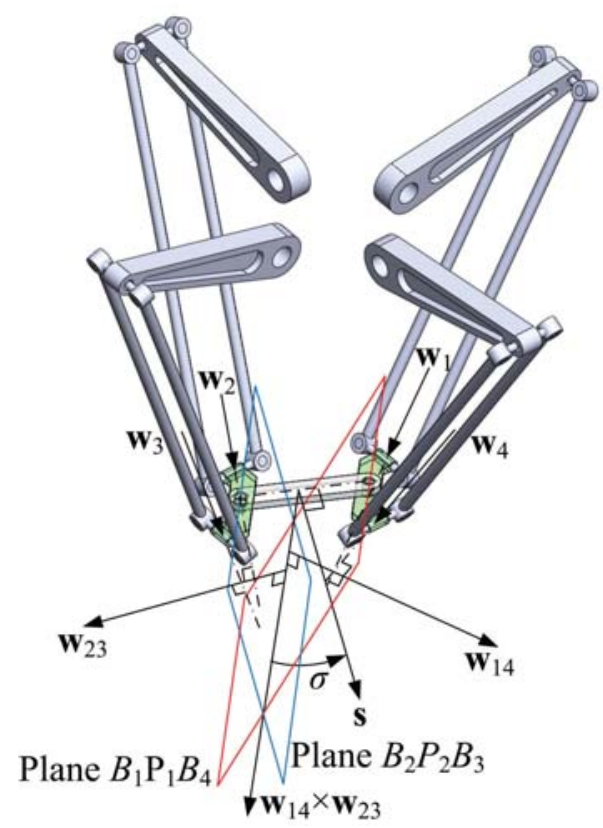

(b)

Figure 3: The pressure angles of the four-limb robots in the motion/force transmission: (a) $\mu_{i}$ for all robots; (b) $\sigma$ for Quattro.

The determinant $|\mathbf{B}|$ of the inverse Jacobian matrix $\mathbf{B}$ takes the form

$$
|\mathbf{B}|=\prod_{i=1}^{4} h_{i}=b^{4} \prod_{i=1}^{4} \mathbf{w}_{i}^{T}\left(\mathbf{u}_{i} \times \mathbf{v}_{i}\right)
$$

subsequently, the pressure angle $\mu_{i}$ associated with the motion transmission in the $i$ th limb, i.e., the motion transmitted from the actuated link to the parallelogram, is defined as:

$$
\mu_{i}=\cos ^{-1}\left(\mathbf{w}_{i}^{T}\left(\mathbf{u}_{i} \times \mathbf{v}_{i}\right)\right), \quad i=1, \ldots, 4
$$

namely, the pressure angle between the velocity of point $B_{i}$ along the vector of $\mathbf{u}_{i} \times \mathbf{v}_{i}$ and the pure force applied to the parallelogram along $\mathbf{w}_{i}$, as shown in Fig. 3(a).

Likewise, while using the Laplace expansion, the determinant $|\mathbf{A}|$ of the forward Jacobian matrix 
Table 2: The determinant of the forward Jacobian matrix for the four-limb robots.

\begin{tabular}{llll}
\hline robots & Quattro (H4) & V4 & Veloce. \\
\hline$|\mathbf{A}|$ & $2 r\left(\mathbf{w}_{14} \times \mathbf{w}_{23}\right)^{T} \mathbf{s}$ & $2 r\left(\mathbf{w}_{14} \times \mathbf{w}_{23}\right)^{T} \mathbf{s}$ & $h \phi /(2 \pi)\left(\mathbf{w}_{13} \times \mathbf{w}_{24}\right)^{T} \mathbf{k}$ \\
\hline \hline
\end{tabular}

A for the Quattro robot is expressed as:

$$
\begin{aligned}
|\mathbf{A}| & =\left|\begin{array}{cc}
\mathbf{w}_{1}^{T} & r \mathbf{w}_{1}^{T} \mathbf{s} \\
\mathbf{w}_{2}^{T} & -r \mathbf{w}_{2}^{T} \mathbf{s} \\
\mathbf{w}_{3}^{T} & -r \mathbf{w}_{3}^{T} \mathbf{s} \\
\mathbf{w}_{4}^{T} & r \mathbf{w}_{4}^{T} \mathbf{s}
\end{array}\right|=\left(-r \mathbf{w}_{1}^{T} \mathbf{s}\left|\begin{array}{r}
\mathbf{w}_{2}^{T} \\
\mathbf{w}_{3}^{T} \\
\mathbf{w}_{4}^{T}
\end{array}\right|+r \mathbf{w}_{4}^{T} \mathbf{s}\left|\begin{array}{c}
\mathbf{w}_{1}^{T} \\
\mathbf{w}_{2}^{T} \\
\mathbf{w}_{3}^{T}
\end{array}\right|\right)+\left(-r \mathbf{w}_{2}^{T} \mathbf{s}\left|\begin{array}{r}
\mathbf{w}_{1}^{T} \\
\mathbf{w}_{3}^{T} \\
\mathbf{w}_{4}^{T}
\end{array}\right|+r \mathbf{w}_{3}^{T} \mathbf{s}\left|\begin{array}{c}
\mathbf{w}_{1}^{T} \\
\mathbf{w}_{2}^{T} \\
\mathbf{w}_{4}^{T}
\end{array}\right|\right) \\
& =r\left(\mathbf{w}_{1} \times \mathbf{w}_{4}\right) \times\left(\mathbf{w}_{2} \times \mathbf{w}_{3}\right) \cdot \mathbf{s}-r\left(\mathbf{w}_{2} \times \mathbf{w}_{3}\right) \times\left(\mathbf{w}_{1} \times \mathbf{w}_{4}\right) \cdot \mathbf{s} \\
& =2 r\left(\mathbf{w}_{1} \times \mathbf{w}_{4}\right) \times\left(\mathbf{w}_{2} \times \mathbf{w}_{3}\right) \cdot \mathbf{s}=2 r\left(\mathbf{w}_{14} \times \mathbf{w}_{23}\right)^{T} \mathbf{s}
\end{aligned}
$$

where $\mathbf{w}_{m n}=\mathbf{w}_{m} \times \mathbf{w}_{n}$, and $\mathbf{s}$ are given in Table 1. By the same token, the expressions of $|\mathbf{A}|$ for the robots under study can be calculated as listed in Table 2, where the corresponding vectors $\mathbf{s}$ are listed in Table 1.

Taking the Quattro robot as an example, the pressure angle $\sigma$ amongst limbs, namely, the force transmitted from the end-effector to the passive parallelograms in the other limbs, provided that the actuated joints in these limbs are locked, is derived below:

$$
\sigma=\cos ^{-1} \frac{\left(\mathbf{w}_{14} \times \mathbf{w}_{23}\right)^{T} \mathbf{s}}{\left\|\mathbf{w}_{14} \times \mathbf{w}_{23}\right\|}
$$

wherefrom the geometrical meaning of angle $\sigma$ can be interpreted as the angle between the minus $Y$-axis (s is normal to segment $\overline{P_{1} P_{2}}$ ) and the intersection line of planes $B_{1} P_{1} B_{4}$ and $B_{2} P_{2} B_{3}$, where plane $B_{1} P_{1} B_{4}\left(B_{2} P_{2} B_{3}\right)$ is normal to the common perpendicular line between the two skew lines along $\mathbf{w}_{1}$ and $\mathbf{w}_{4}\left(\mathbf{w}_{2}\right.$ and $\left.\mathbf{w}_{3}\right)$, as depicted in Fig. 3(b). To illustrate the angle $\sigma$ physically, $\left(\mathbf{w}_{14} \times \mathbf{w}_{23}\right)^{T} \mathbf{s}$ can be rewritten in the following form:

$$
\left(\mathbf{w}_{14} \times \mathbf{w}_{23}\right)^{T} \mathbf{s}=\mathbf{w}_{14}^{T}\left[\mathbf{w}_{3}\left(\mathbf{w}_{2} \cdot \mathbf{s}\right)-\mathbf{w}_{2}\left(\mathbf{w}_{3} \cdot \mathbf{s}\right)\right]=\mathbf{w}_{23}^{T}\left[\mathbf{w}_{4}\left(\mathbf{w}_{1} \cdot \mathbf{s}\right)-\mathbf{w}_{1}\left(\mathbf{w}_{4} \cdot \mathbf{s}\right)\right]
$$

The angle $\sigma$ now can be interpreted as the pressure angle between the velocity in the direction of $\mathbf{w}_{1} \times \mathbf{w}_{4}$ and the forces along $\mathbf{w}_{2} \times \mathbf{w}_{3}$ imposed by the parallelograms in limbs 2 and 3 to point $P$, 
under the assumption that the actuated joints in limbs 1 and 4 are locked simultaneously. The same explanation is applicable for the case when the actuated joints in limbs 2 and 3 are locked.

By the same token, the pressure angle for the remaining robot counterparts can be defined. Consequently, the motion $\kappa$ and force $\zeta$ transmission indices (TI) in a prescribed configuration are defined as the minimum value of the cosine of the pressure angles, respectively,

$$
\kappa=\min \left(\left|\cos \mu_{i}\right|\right), i=1, \ldots, 4 ; \quad \zeta=|\cos \sigma|
$$

To this end, the local transmission index (LTI) [38] is defined as

$$
\eta=\min \{\kappa, \zeta\}=\min \left\{\left|\cos \mu_{i}\right|,|\cos \sigma|\right\} \in[0,1]
$$

The larger the value of $\eta$, the better the transmission quality of the manipulator.

\subsection{Singularity Analysis}

It is known that the manipulator reaches a singularity when the determinant of its forward or/and the determinant of its inverse Jacobian matrix is (are) equal to zero [44]. Henceforth, the manipulator singularities are analyzed by resorting to the motion/force transmission, as this approach can effectively evaluate the closeness to a singularity, compared to the Jacobian method $[44,48]$ and Grassmann Geometry [45] or Grassmann-Cayley Algebra [46]. For the Quattro robot, apparently, Eqs. (11) and (13) show that:

- the type-1 singularity, also named limb singularity, occurs when $\mu_{i}=90^{\circ}$, namely, $|\mathbf{B}|=0$;

- the type-2 singularity, also named actuation singularity $[47,49]$, occurs when $\sigma=90^{\circ}$, leading to $|\mathbf{A}|=0$.

The pressure angles $\mu_{i}$ and $\sigma$ are now employed to describe the type- 1 and type- 2 singularities.

Figure 4 shows the type- 1 singularities, i.e., the actuated link $A_{i} B_{i}$ and the segment of parallelogram $B_{i} C_{i}$ being coplanar. Type-1 singularities usually exist at the reachable workspace boundaries, outside the regular operational workspace, which can be readily identified. In this work, we will focus on the type-2 singularity.

Figure 5 shows four typical Type- 2 singularities with $\sigma=90^{\circ}$ or $\zeta=|\cos \sigma|=0[5,50]$ :

- Figure 5(a) shows a singular configuration corresponding to $\mathbf{w}_{1}$ being parallel to $\mathbf{w}_{4}$, which means 


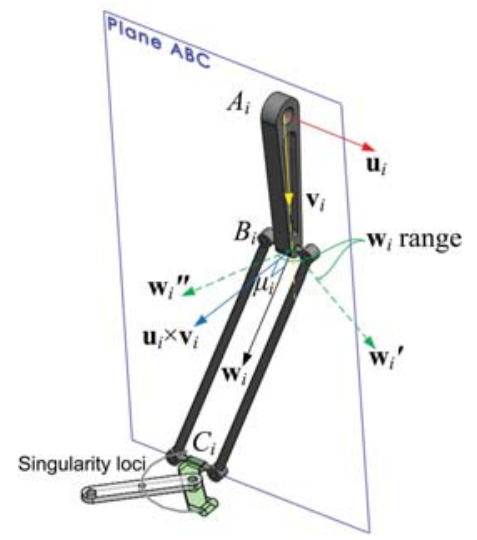

(a)
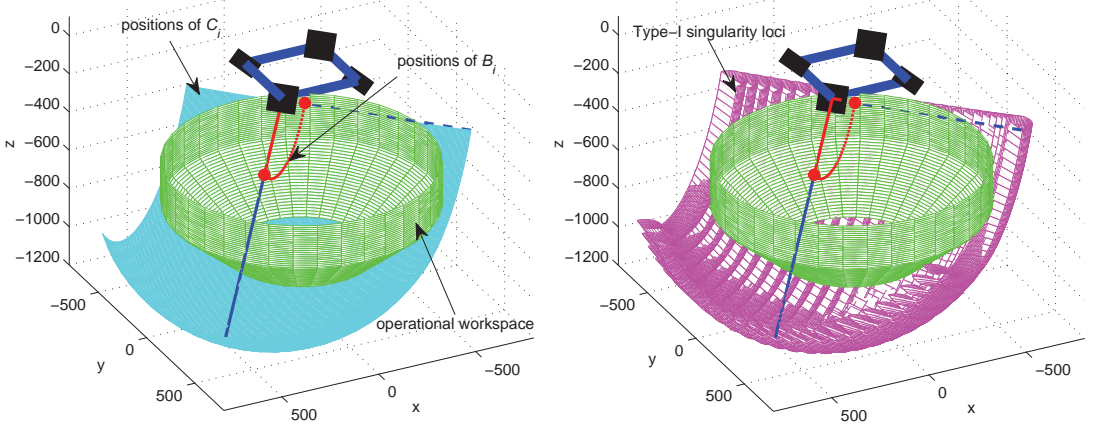

(b)

Figure 4: The type-1 singularity of the $i$ th limb with $\phi \in[-\pi / 2, \pi / 2]$ : (a) limb in a singular configuration with coplanar vectors $\mathbf{u}_{i}, \mathbf{v}_{i}, \mathbf{w}_{i}$; (b) singularity loci.

that $\mathbf{w}_{14}=\mathbf{w}_{1} \times \mathbf{w}_{4}=\mathbf{0}$ becomes a zero vector, allowing the robot to have extra mobilities even though all the actuated joints are locked, i.e., the constraint singularity.

- Figure 5(b) shows an actuation singularity $\mathbf{w}_{14}$ parallel to $\mathbf{w}_{23}$, leading to the vanished 2-DOF motions due to the linear dependency of the two vectors.

- Figure 5(c) shows an actuation singularity with $\mathbf{w}_{14}$ parallel to $\mathbf{s}$, wherein the robot loses 1-DOF motion.

- Figure 5(d) shows another parallel singularity in the case of the general linear complex. The wrench exerting on the end-effector is generated by one of the transmission wrenches that is proportional to those that are generated by the other transmission wrenches, thus, the robot loses 1-DOF motion in this singular configuration.

Based on the above analysis, the transmission index defined in Eq. (16) can be used for the evaluation of singularity and closeness to a singularity, where a large value for $\eta$ means that the robot is far away from singular configurations. The singularity occurs when $\eta=0$. When $\eta \leq \epsilon$, $0<\epsilon \ll 1$ being a computational tolerance, the robot is considered to be in a singular configuration. It can also effectively measure the closeness to a singularity that cannot be found by the Grassmman algorithm based singularity analysis, with the index's value bounded by $[0,1]$. Moreover, this method can efficiently evaluate the closeness to a singularity numerically, compared to the conditioning of the normalized Jacobian matrix by a characteristic length due to the dimensional inhomogeneity, as the latter significantly depends on the selection of the characteristic length that is not unique. 


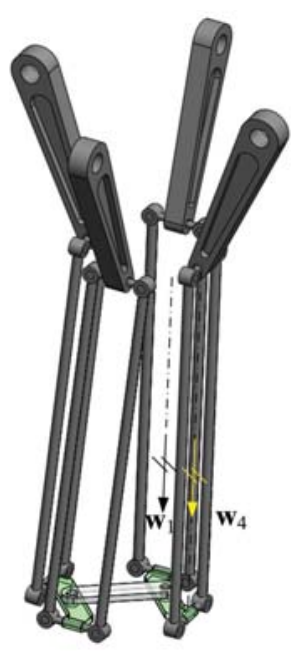

(a)

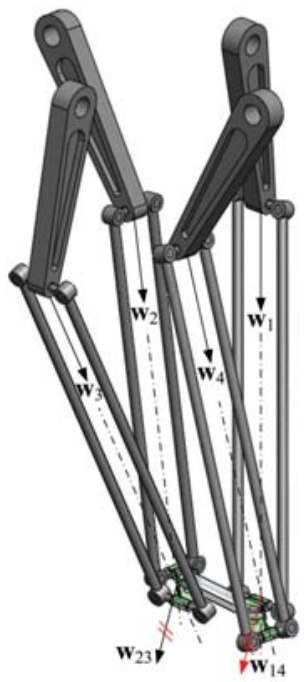

(b)

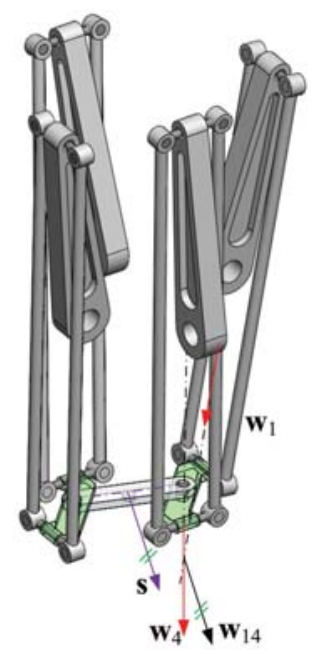

(c)

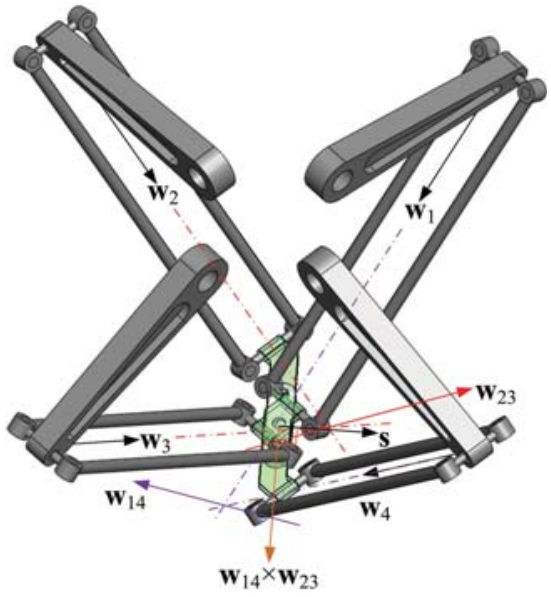

(d)

Figure 5: Four cases of the Type-2 singularities of the Quattro robot: (a) $\mathbf{w}_{1} \| \mathbf{w}_{4}\left(\mathbf{w}_{2} \| \mathbf{w}_{3}\right)$; (b) $\mathbf{w}_{14}\left\|\mathbf{w}_{23} ;(\mathrm{c}) \mathbf{w}_{14}\left(\mathbf{w}_{23}\right)\right\| \mathbf{s} ;(\mathrm{d})\left(\mathbf{w}_{14} \times \mathbf{w}_{23}\right) \perp \mathbf{s}$.

Table 3: Geometrical parameters of the base and mobile platform of the four-limb robots.

\begin{tabular}{lll}
\hline \hline robots & base & mobile platform \\
\hline Quattro & $\alpha=-\pi / 4, \beta=3 \pi / 4$ & $r=80 \mathrm{~mm}, c=70 \mathrm{~mm}$ \\
H4, V4 & $\alpha=0, \beta=\pi / 2$ & $r=80 \mathrm{~mm}, c=70 \mathrm{~mm}$ \\
Veloce. & $\alpha=-\pi / 4, \beta=3 \pi / 4$ & $r=100 \mathrm{~mm}, \gamma=(2 i-1) \pi / 4, h$ \\
\hline \hline
\end{tabular}

\section{Case Studies-Performance Evaluation of PnP Robots}

In this section, the motion/force transmission and singularities of the Quattro, H4, Veloce. and V4 robots are analyzed. According to the technical parameters of the Quattro robot [11], the parameters of the robots' base and mobile platforms are given in Table 3 , and other parameters are set to $R=$ $275 \mathrm{~mm}, b=375 \mathrm{~mm}$ and $l=800 \mathrm{~mm}$, respectively. The operational WS of the robots are shown in Fig. 6.

\subsection{Pressure Angles Along a Spiral Trajectory}

To illustrate the previously defined pressure angles, the Jacobian determinants and the pressure angles of the Quattro robot along a spiral trajectory within the operational workspace are plotted in Fig. 7. The spiral path is expressed as:

$$
x=650 \rho \cos \vartheta ; \quad y=650 \rho \sin \vartheta ; \quad z=-975+500 \rho ; \quad \phi=\pi \rho-\pi / 2
$$




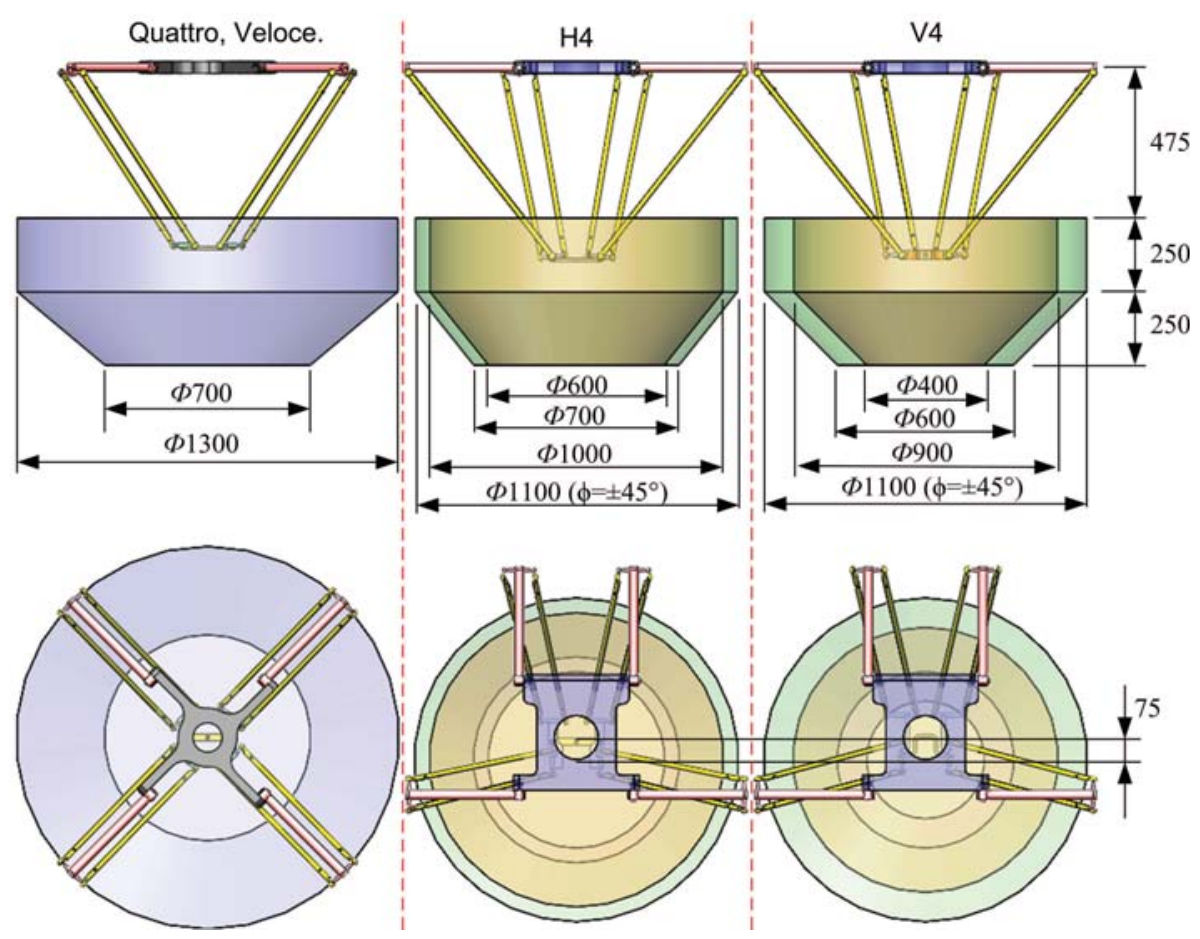

Figure 6: The operational workspace of the robots with rotational capability of $\phi= \pm 90^{\circ}$.

with

$$
\rho=\vartheta /(4 \pi) ; \quad \vartheta \in[0,4 \pi]
$$

It is noteworthy that the robot encounters Type-2 singularity when $|\phi|=\pi / 2$ at the end points of the path, as shown in Fig. 7(b). On the other hand, the pressure angle $\sigma$ is equal to zero, i.e., the transmission quality of force transmission is the best, when $\phi=0$. Moreover, the pressure angles $\mu_{i}$ are in the range of $\left[0,65^{\circ}\right]$, meaning that the robot is far from the type- 1 singularity, which is consistent with the varying of the determinants of inverse Jacobian.

\subsection{Isocontours of the Transmission Index}

The LTI isocontours of the four robots under consideration are visualized in Figs. 8 to 11, together with the max-/minimum LTI within the operational workspace listed in Table 4 . It is seen that the minimum LTI of the Quattro and Veloce. robots are much higher than those of H4 and V4 robots. Moreover, the volume of the working area where LTI is greater than 0.7, namely, where the transmission quality is good, is larger with the Quattro and Veloce. robots than with the H4 and V4 robots. This means that the four-limb robots with a fully symmetrical architecture have better transmission performance than their counterparts with an asymmetric one. It should be also noted 


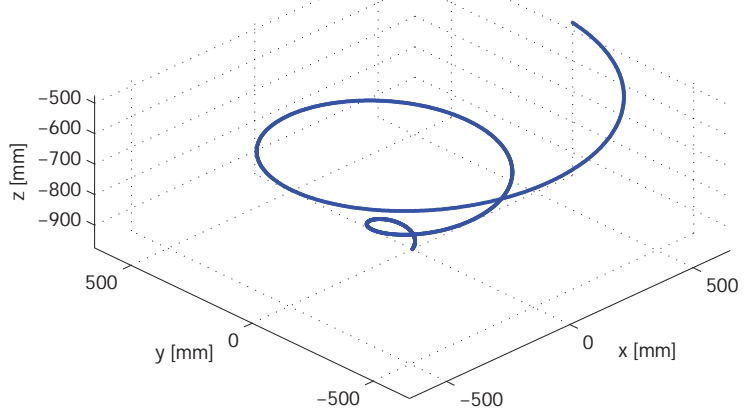

(a)
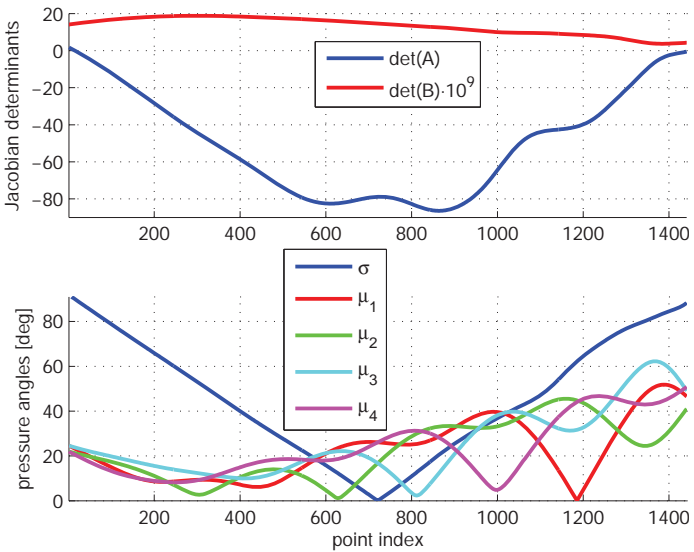

(b)

Figure 7: The Jacobian determinants and pressure angles along a spiral line: (a) trajectory; (b) determinants and pressure angles.

Table 4: The min-/maximum LTI within the operational workspace.

\begin{tabular}{l|ll|ll|ll}
\hline \hline robots & \multicolumn{2}{|l|}{$\phi=0$} & & \multicolumn{2}{l|}{$\phi=45^{\circ}$} & \multicolumn{2}{l}{$\phi=360^{\circ}(h=20 \mathrm{~mm})$} \\
\hline LTI & $\eta_{\min }$ & $\eta_{\max }$ & $\eta_{\min }$ & $\eta_{\max }$ & $\eta_{\min }$ & $\eta_{\max }$ \\
\hline Quattro & 0.380 & 1.0 & 0.173 & 0.750 & - & - \\
H4 & 0.031 & 0.917 & 0 & 0.847 & - & - \\
Veloce. & 0.287 & 1.0 & - & - & 0.268 & 0.999 \\
V4 & 0.008 & 0.872 & 0 & 0.804 & - & - \\
Ragnar & 0.238 & 0.892 & - & - & 0.175 & 0.885 \\
\hline \hline
\end{tabular}

that the higher the rotation angle of the moving-platform, the lower the transmission performance of the robots.

As displayed in Fig. 8, the transmission index of the Quattro robot has larger values in the central region, which admits a singularity-free workspace with rotational capability $\phi= \pm 45^{\circ}$. Similarly, Fig. 9 shows that the Veloce. robot can also have a high-transmission workspace free of singularity with smaller lead of screw pair, which means that this type of mobile platform allows the robot to have high performance in terms of transmission quality and a large orientation workspace. On the contrary, the asymmetric $\mathrm{H} 4$ and V4 robots result in relatively small operational workspace and relatively low transmission performance, as shown in Figs. 10 and 11, but with similar mechanism footprint ratio.

On the basis of the previous comparison, let's investigate transmission performance of the recently reported 'Ragnar' robot [15] with the mobile platform of Veloce. robot. This four-limb robot adopts an asymmetrical architecture, as depicted in Fig. 12, which admits a long and narrow workspace for the efficient use of the shop-floor space. The Cartesian coordinates of points $A_{i}, i=1, \ldots, 4$, are 


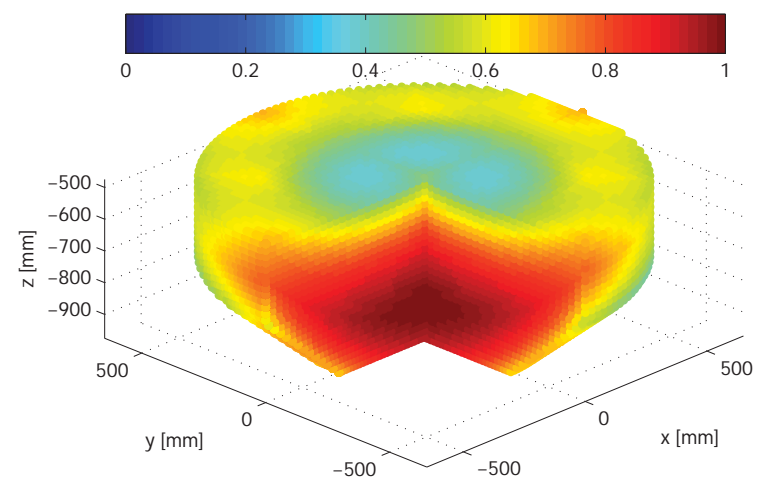

(a)

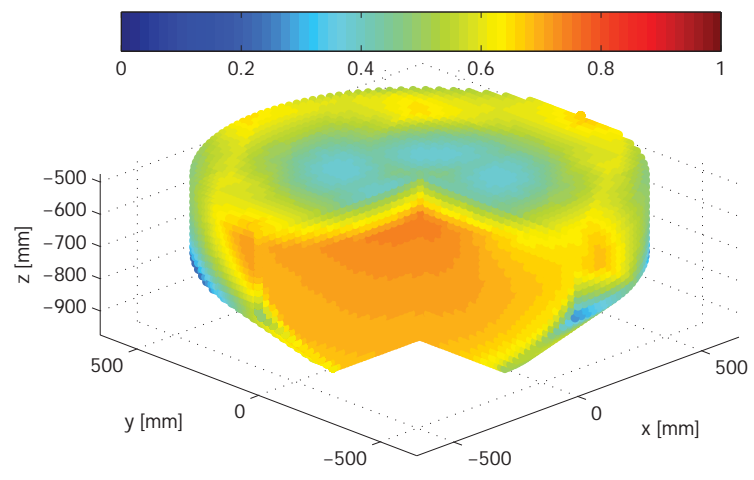

(b)

Figure 8: The LTI isocontours of the Quattro robot: (a) $\phi=0$; (b) $\phi=45^{\circ}$.

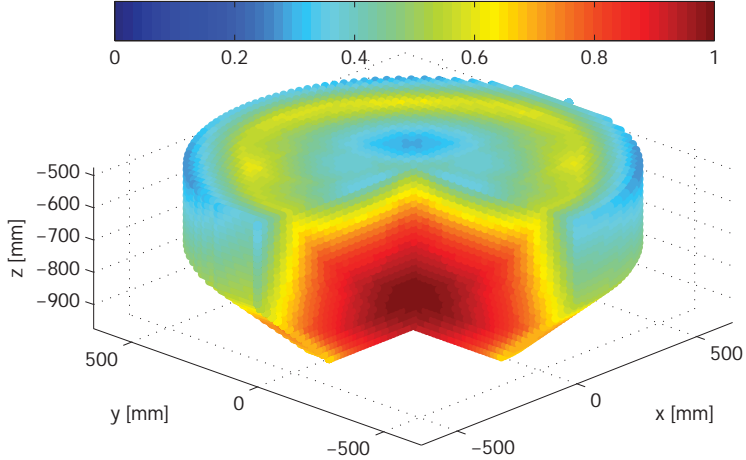

(a)

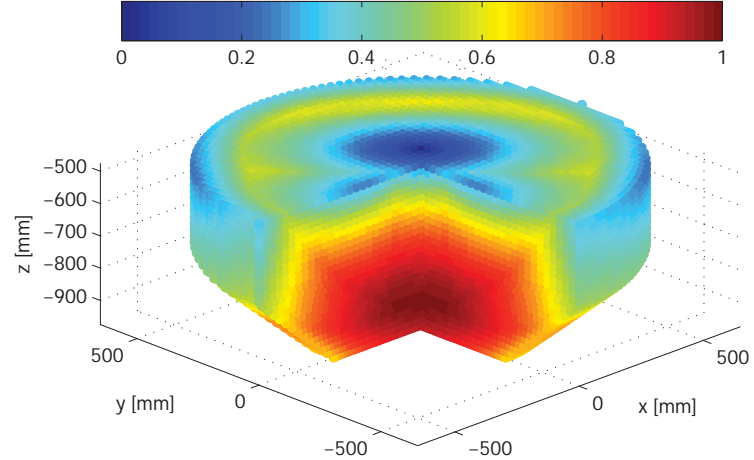

(b)

Figure 9: The LTI isocontours of the Veloce. robot with $\phi=2 \pi$ : (a) screw lead $h=20 \mathrm{~mm}$; (b) screw lead $h=50 \mathrm{~mm}$.

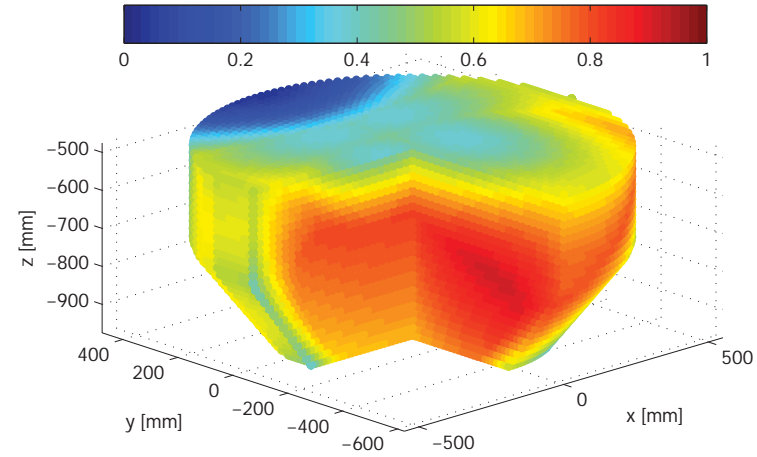

(a)

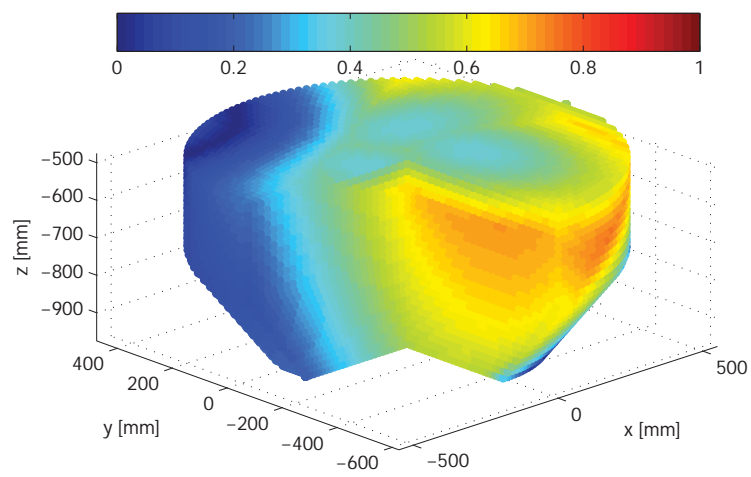

(b)

Figure 10: The LTI isocontours of the H4 robot: (a) $\phi=0$; (b) $\phi=45^{\circ}$. 


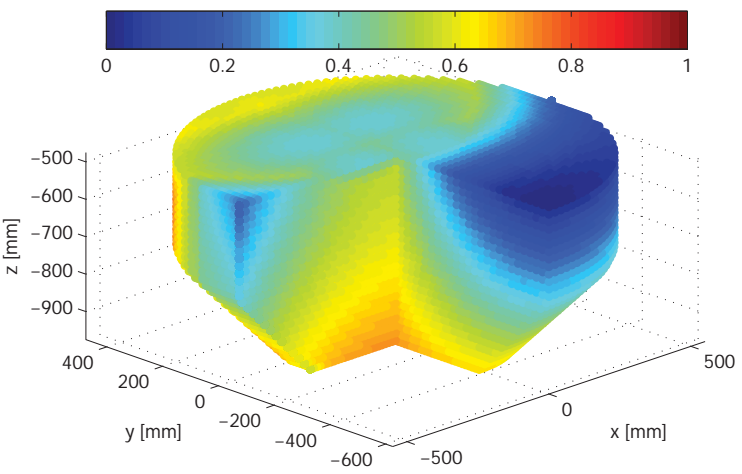

(a)

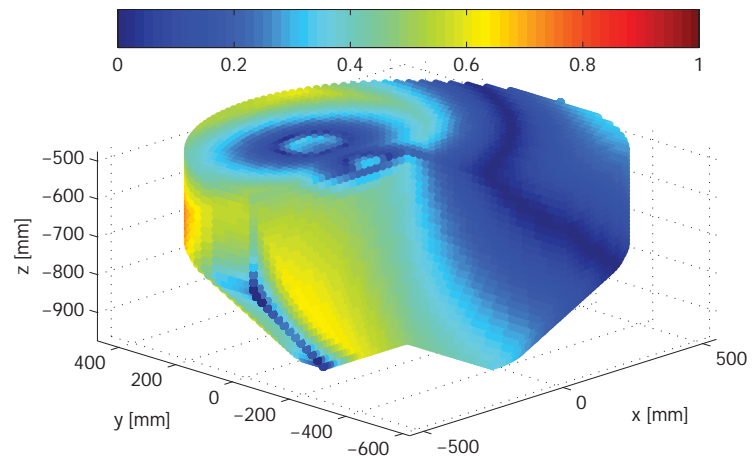

(b)

Figure 11: The LTI isocontours of the V4 robot: (a) $\phi=0$; (b) $\phi=45^{\circ}$.

expressed as in the base frame as $\mathbf{a}_{1}=-\mathbf{a}_{3}=\left[\begin{array}{lll}350 & 100 & 0\end{array}\right]^{T}, \mathbf{a}_{2}=-\mathbf{a}_{4}=\left[\begin{array}{lll}-350 & 100 & 0\end{array}\right]^{T}$, and the axis of rotation $\mathbf{u}_{i}$ of the $i$ th actuated joint is parallel to the $x-z$ plane. Other parameters are identical to the Veloce. robot. With these parameters, the maximum regular workspace of the robot is approximately fitted to a super-ellipsoid defined mathematically as:

$$
\left|\frac{x}{505}\right|^{3}+\left|\frac{y}{685}\right|^{3}+\left|\frac{z+575}{365}\right|^{3}=1
$$

the corresponding LTI isocontours over the workspace are displayed in Fig. 13 sequentially with the max-/minimum LTI in Table 4, from which it is seen that this platform can also make the Ragnar robot have a high-transmission workspace free of singularity. This new robot configuration performs high transmission quality with high rotational capability, particularly, a rectangular workspace suitable for pick-and-place operations.

\subsection{Closeness to Actuation Singularity}

With aid of the transmission analysis, for instance, the LTI distributions in Figs. 8 to 11, numerical evaluation of singularity or closeness to a singularity is applicable. Based upon the analysis, this type of robots is free of constraint singularity under the predefined working mode in Sec. 3. Figures 14 to 16 illustrate the actuation singularity loci of the robots when $\zeta=|\cos \sigma| \leq \epsilon$, with $\epsilon=0.001$, where the volumes bounded by the blue grids stand for the operational workspace and the colored surfaces depict for the singularity loci.

It is seen that the singularity surfaces of the robots become larger with the increasing rotation angle, moving towards the central region from the workspace boundaries. The orientations of the 


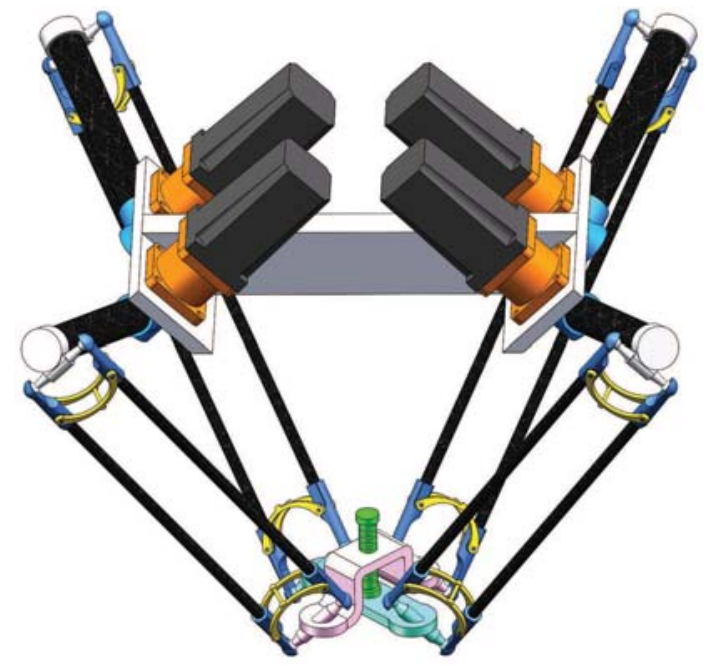

(a)

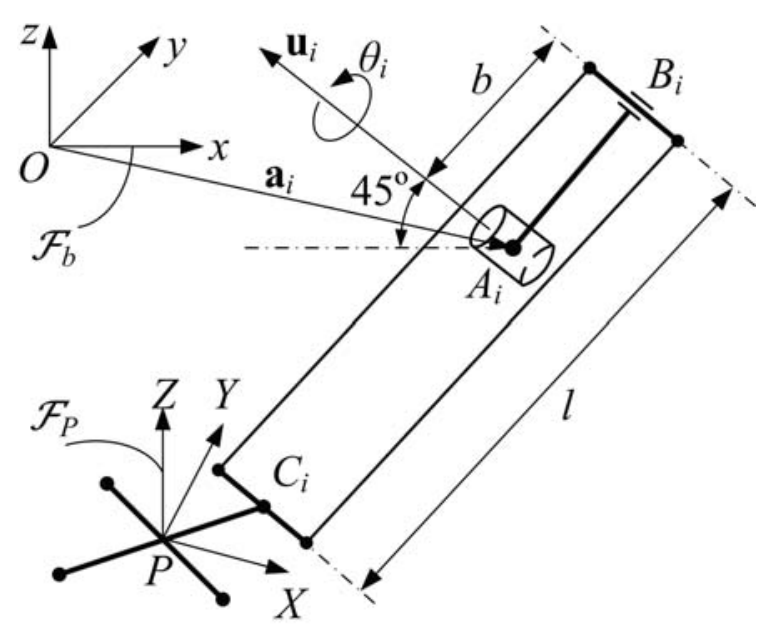

(b)

Figure 12: The asymmetrical Ragnar robot: (a) CAD model; (b) parameterization of the $i$ th limb.

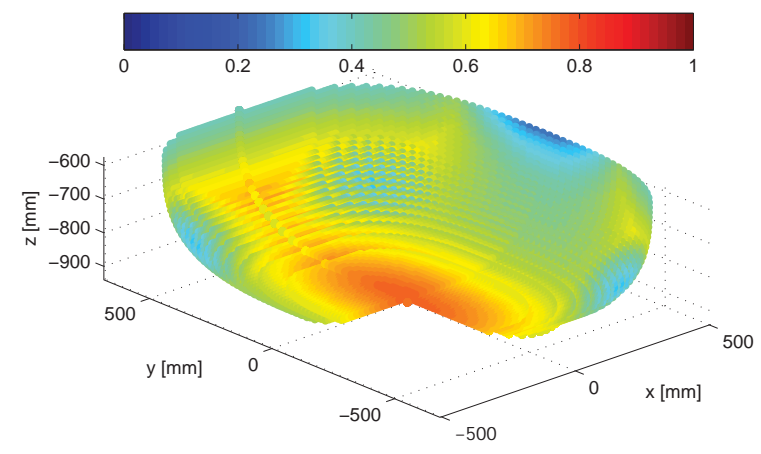

(a)

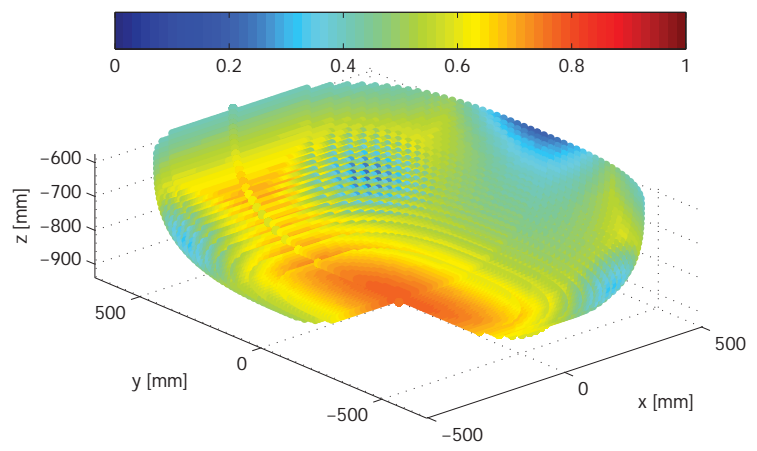

(b)

Figure 13: The LTI isocontours of the Ragnar robot with the screw-paired mobile platform of lead $h=20$ : (a) $\phi=0$; (b) $\phi=2 \pi$. 


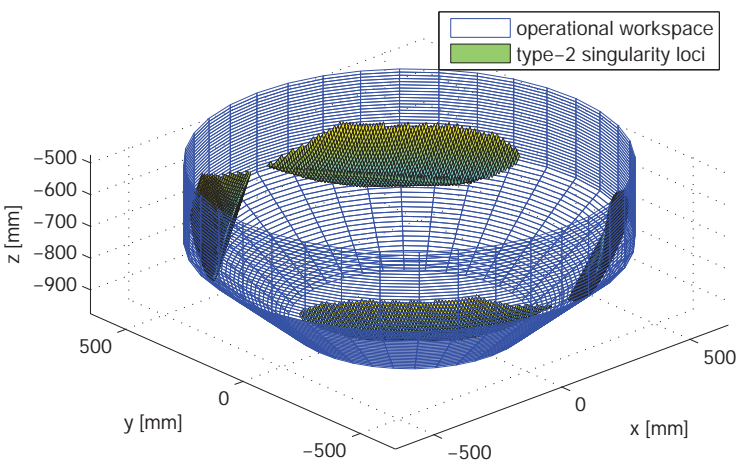

(a)

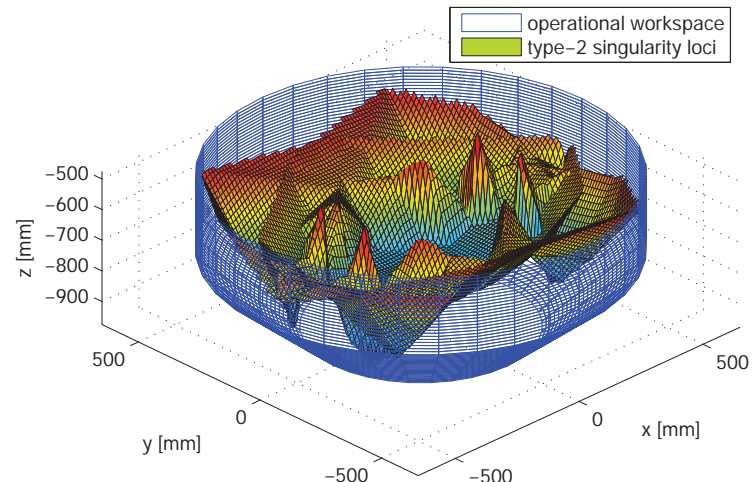

(b)

Figure 14: Actuation singularity loci of Quattro: (a) $\phi= \pm 75^{\circ}$; (b) $\phi= \pm 90^{\circ}$.

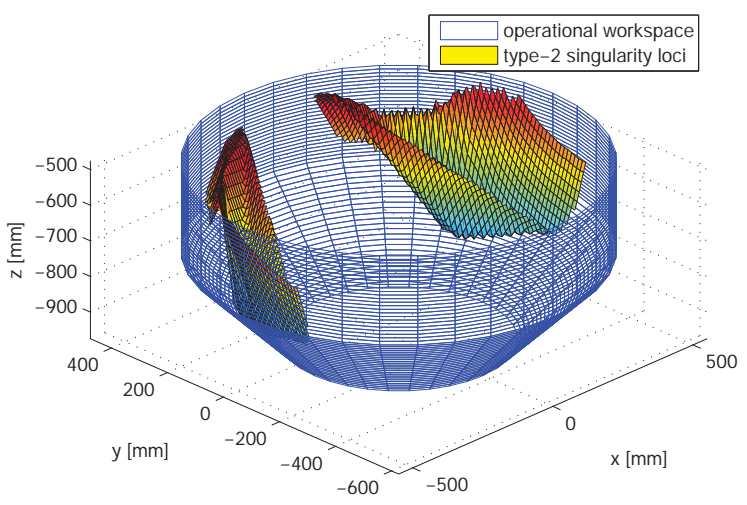

(a)

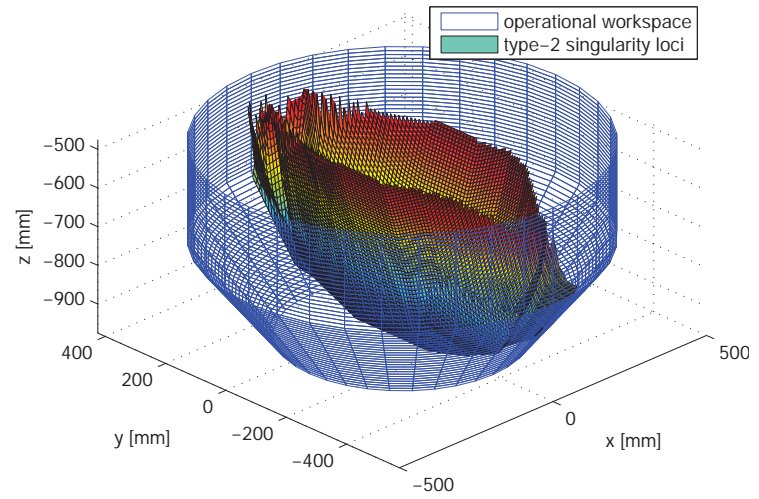

(b)

Figure 15: Actuation singularity loci of H4: (a) $\phi= \pm 60^{\circ}$; (b) $\phi= \pm 90^{\circ}$.

robots with the articulated mobile platforms are limited to $\pm 90^{\circ}$ in practice. Moreover, the Quattro robot still admits a large singularity-free workspace with rotational capability $\phi= \pm 75^{\circ}$, while the V4 robot has the smallest dexterous workspace with the increasing rotation angle. On the other hand, the $\mathrm{V} 4$ robot can be mounted vertically to save space.

\section{Conclusions}

This paper presents a uniform evaluation method for the transmission quality and singularity analysis for a class of four-limb parallel Schönflies-motion robots with articulated mobile platforms, which are closely in connection with two pressure angles derived from the forward and inverse Jacobian matrices. The cosine function of the pressure angles based indices are defined to evaluate the transmission quality. Moreover, the two pressure angles can be used efficiently to measure the transmission quality of four- 


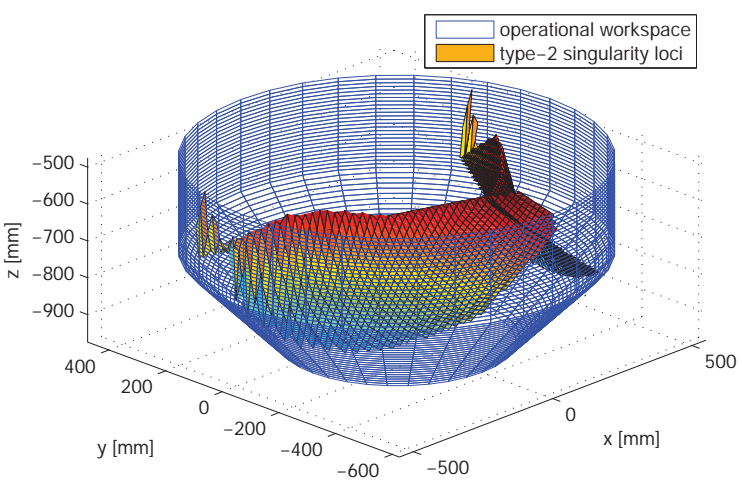

(a)

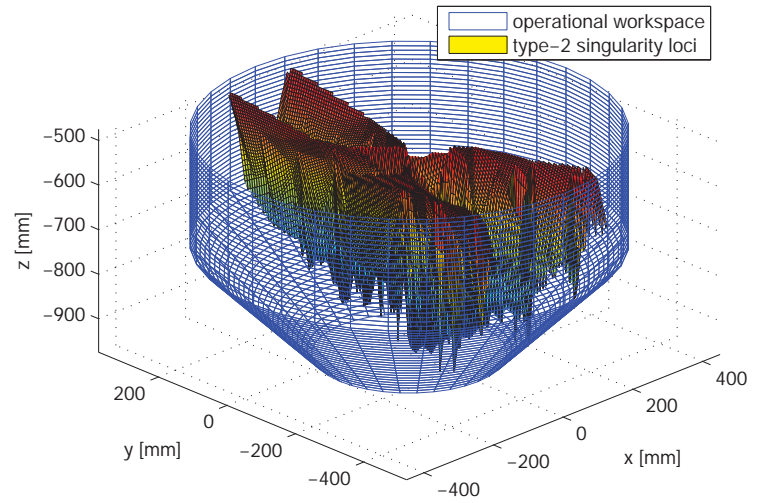

(b)

Figure 16: Actuation singularity loci of V4: (a) $\phi= \pm 45^{\circ}$; (b) $\phi= \pm 90^{\circ}$.

limb parallel Schönflies-motion robots. It is noteworthy that the pressure angles are symbolically formulated and the transmission indices are bounded between 0 and 1 .

The proposed method is applied to five robot counterparts, where critical rotation angles, i.e., $75^{\circ}, 60^{\circ}$ and $45^{\circ}$, are identified for three of them with the end-effectors connected to the two subplatforms by two revolute joints, showing their constant-orientation translational workspace with different rotational capabilities. Finally, it is shown that robots with the screw-pair-based mobile platform, i.e., the Veloce. robot and the new configuration of the asymmetrical Ragnar robot, lead to high transmission quality and large operational workspace free of singularity.

\section{Acknowledgement}

This work was partly supported by the Doctoral Scientific Research Foundation of Liaoning Province (No. 20170520134) and Innovation Fund Denmark (137-2014-5). The support from Aalborg University and Blue Workforce A/S is gratefully acknowledged.

\section{References}

[1] S. Krut, V. Nabat, O. Company, and F. Pierrot. A high-speed parallel robot for SCARA motions. In IEEE Int. Conf. Robot. Autom., volume 4, pages 4109-4115, April 2004.

[2] F. Pierrot, V. Nabat, O. Company, S. Krut, and P. Poignet. Optimal design of a 4-dof parallel manipulator: From academia to industry. IEEE Trans. Robot., 25(2):213-224, 2009. 
[3] S. M. Kim, W. Kim, and B.-J. Yi. Kinematic analysis and optimal design of a 3T1R type parallel mechanism. In IEEE Int. Conf. Robot. Autom., pages 2199-2204, 2009.

[4] O. Altuzarra, B. Şandru, Ch. Pinto, and V. Petuya. A symmetric parallel Schönflies-motion manipulator for pick-and-place operations. Robotica, 29:853-862, 2011.

[5] S. Liu, T. Huang, J. Mei, X. Zhao, P. Wang, and D. G Chetwynd. Optimal design of a 4-DOF SCARA type parallel robot using dynamic performance indices and angular constraints. ASME J. Mech. Robot., 4(3):031005, 2012.

[6] G. Wu, S. Bai, and P. Hjørnet. On the stiffness of three/four degree-of-freedom parallel pickand-place robots with four identical limbs. In IEEE Int. Conf. Robot. Autom., pages 861-866, Stockholm, Sweden, 2016.

[7] F. Pierrot and O. Company. H4: a new family of 4-dof parallel robots. In IEEE/ASME Inter. Conf. Advan. Intell. Mecha., pages 508-513. IEEE, 1999.

[8] O. Company, S. Krut, and F. Pierrot. Internal singularity analysis of a class of lower mobility parallel manipulators with articulated traveling plate. IEEE Trans. Robot., 22(1):1-11, 2006.

[9] S. Krut, M. Benoit, H. Ota, and F. Pierrot. I4: A new parallel mechanism for SCARA motions. In IEEE Int. Conf. Robot. Autom., volume 2, pages 1875-1880. IEEE, 2003.

[10] V. Nabat, M. de la O Rodriguez, O. Company, S. Krut, and F. Pierrot. Par4: very high speed parallel robot for pick-and-place. In IEEE/RSJ Inter. Conf. Intell. Robot. Syst., pages 553-558. IEEE, 2005.

[11] Adept Quattro Parallel Robots. http://www1.adept.com/main/ke/data/Archived/Quattro/sQuattro_UG.pdf.

[12] Penta Veloce. http://pentarobotics.com/products/\#brochure.

[13] O. Salgado, O. Altuzarra, V. Petuya, and A. Hernández. Synthesis and design of a novel 3T1R fully-parallel manipulator. ASME J. Mech. Des., 130(4):042305, 2008.

[14] F. Xie and X. Liu. Design and development of a high-speed and high-rotation robot with four identical arms and a single platform. ASME J. Mech. Robot., 7(4):041015, 2015.

[15] G. Wu, S. Bai, and P. Hjørnet. Architecture optimization of a parallel Schönflies-motion robot for pick-and-place applications in a predefined workspace. Mech. Mach. Theory, 106:148-165, 2016. 
[16] X. Kong and C. M. Gosselin. Type synthesis of 3T1R 4-dof parallel manipulators based on screw theory. IEEE Trans. Robot. Autom., 20(2):181-190, 2004.

[17] G. Gogu. Structural synthesis of fully-isotropic parallel robots with Schönflies motions via theory of linear transformations and evolutionary morphology. Euro. J. Mech.-A/Solids, 26(2):242-269, 2007.

[18] P.-L. Richard, C. M. Gosselin, and X. Kong. Kinematic analysis and prototyping of a partially decoupled 4-DOF 3T1R parallel manipulator. ASME J. Mech. Des., 129(6):611-616, 2007.

[19] Q. Li and J. M. Hervé. Parallel mechanisms with bifurcation of Schoenflies motion. IEEE Trans. Robot., 25(1):158-164, 2009.

[20] S. Briot, V. Arakelian, and S. Guégan. PAMINSA: A new family of partially decoupled parallel manipulators. Mech. Mach. Theory, 44(2):425-444, 2009.

[21] F. Pierrot, C. Reynaud, and A. Fournier. DELTA: a simple and efficient parallel robot. Robotica, 8(2):105-109, 1990.

[22] J. Angeles, S. Caro, W. Khan, and A. Morozov. Kinetostatic design of an innovative Schönfliesmotion generator. Proc. Ins. Mech. Eng. Part C: J. Mech. Eng. Sci., 220(7):935-943, 2006.

[23] P.-C. Lee and J.-J. Lee. Singularity and workspace analysis of three isoconstrained parallel manipulators with schoenflies motion. Front. Mech. Eng., 7(2):163-187, 2012.

[24] T. Harada and J. Angeles. Kinematics and singularity analysis of a CRRHHRRC parallel Schönflies motion generator. CSME Transactions, 38(2):173-183, 2014.

[25] S. M. Kim, K. Shin, B.-J. Yi, and W. Kim. Development of a novel two-limbed parallel mechanism having schönflies motion. Proc. Ins. Mech. Eng. Part C: J. Mech. Eng. Sci., 229(1):136-154, 2015.

[26] G. Wu. Kinematic analysis and optimal design of a wall-mounted four-limb parallel Schönfliesmotion robot for pick-and-place operations. J. Intell. Robot. Syst., 2016.

[27] J.-P. Merlet. Jacobian, manipulability, condition number, and accuracy of parallel robots. ASME J. Mech. Des., 128(1):199-206, 2006.

[28] S.-G. Kim and J. Ryu. New dimensionally homogeneous Jacobian matrix formulation by three end-effector points for optimal design of parallel manipulators. IEEE Trans. Robot. Autom., 19(4):731-736, 2003. 
[29] F. Ranjbaran, J. Angeles, M.A. Gonzalez-Palacios, and R. V Patel. The mechanical design of a seven-axes manipulator with kinematic isotropy. J. Intell. Robot. Syst., 14(1):21-41, 1995.

[30] O. Altuzarra, A. Hernandez, O. Salgado, and J. Angeles. Multiobjective optimum design of a symmetric parallel Schönflies-motion generator. ASME J. Mech. Des., 131(3):031002, 2009.

[31] G. Pond and J. A. Carretero. Formulating Jacobian matrices for the dexterity analysis of parallel manipulators. Mech. Mach. Theory, 41(12):1505-1519, 2006.

[32] W. Wei and N. Simaan. Design of planar parallel robots with preloaded flexures for guaranteed backlash prevention. ASME J. Mech. Robot., 2(1):011012, 2010.

[33] J. Angeles. Is there a characteristic length of a rigid-body displacement? Mech. Mach. Theory, 41(8):884-896, 2006.

[34] T.L. Dresner and K.W. Buffinton. Definition of pressure and transmission angles applicable to multi-input mechanisms. ASME J. Mech. Des., 113:495-499, 1991.

[35] S. Bawab, G.L. Kinzel, and K.J. Waldron. Rectified synthesis of six-bar mechanisms with welldefined transmission angles for four-position motion generation. ASME J. Mech. Des., 118:377383, 1996.

[36] S. S Balli and S. Chand. Transmission angle in mechanisms (Triangle in mech). Mech. Mach. Theory, 37(2):175-195, 2002.

[37] Y. Takeda and H. Funabashi. A transmission index for in-parallel wire-driven mechanisms. JSME Inter. J. Series C Mech. Syst., Mach. Elem. Manuf., 44(1):180-187, 2001.

[38] J. Wang, C. Wu, and X. Liu. Performance evaluation of parallel manipulators: Motion/force transmissibility and its index. Mech. Mach. Theory, 45(10):1462-1476, 2010.

[39] X. J. Liu, C. Wu, and J. S. Wang. A new approach for singularity analysis and closeness measurement to singularities of parallel manipulators. ASME J. Mech. Robot., 4(4):041001, 2012.

[40] H. Liu, M. Wang, T. Huang, D. G. Chetwynd, and A. Kecskeméthy. A dual space approach for force/motion transmissibility analysis of lower mobility parallel manipulators. ASME J. Mech. Robot., 7(3):1-7, 2015. 
[41] Y. Zhao, J. Wang, Y. Cao, B. Liang, and T. Zhao. Constant motion/force transmission analysis and synthesis of a class of translational parallel mechanisms. Mech. Mach. Theory, 108:57-74, 2017.

[42] J. Brinker, B. Corves, and Y. Takeda. Kinematic performance evaluation of high-speed delta parallel robots based on motion/force transmission indices. Mech. Mach. Theory, 2018.

[43] H. Liu, T. Huang, A. Kecskeméthy, and D. G Chetwynd. A generalized approach for computing the transmission index of parallel mechanisms. Mech. Mach. Theory, 74:245-256, 2014.

[44] C. Gosselin and J. Angeles. Singularity analysis of closed-loop kinematic chains. IEEE Trans. Robot. Autom., 6(3):281-290, 1990.

[45] J.-P. Merlet. Singular configurations of parallel manipulators and Grassmann geometry. The Int. J. Robot. Res., 8(5):45-56, 1989.

[46] S. Amine, M. T. Masouleh, S. Caro, P. Wenger, and C. Gosselin. Singularity conditions of 3T1R parallel manipulators with identical limb structures. ASME J. Mech. Robot., 4(1):011011, 2012.

[47] S. Amine, O. Mokhiamar, and S. Caro. Classification of 3T1R parallel manipulators based on their wrench graph. ASME J. Mech. Robot., 9(1):011003-1-011003-10, 2017.

[48] G. Wu, S. Caro, S. Bai, and J. Kepler. Dynamic modeling and design optimization of a 3-DOF spherical parallel manipulator. Robot. Auto. Syst., 62(10):1377-1386, 2014.

[49] S. Maraje, L. Nurahmi, and S. Caro. Operation modes comparison of a reconfigurable 3-PRS parallel manipulator based on kinematic performance. In ASME 2016 Int. Design Engineering Technical Conferences and Computers and Information in Engineering Conference, Charlotte, North Carolina, USA, August 21-24 2016.

[50] M. Isaksson, K. Marlow, T. Brogårdh, and A. Eriksson. A comparison of the yaw constraining performance of SCARA-tau parallel manipulator variants via screw theory. In IEEE Int. Conf. Robot. Auto., pages 888-893, 2016. 